\title{
Phonon softening and dispersion in the 1D Holstein model of spinless fermions
}

\author{
C. E. Creffield ${ }^{1}$, G. Sangiovanni ${ }^{2}$, and M. Capone ${ }^{2,3}$ \\ 1 Dipartimento di Fisica, Università di Roma "La Sapienza", Piazzale Aldo Moro 2, I-00185 Rome, Italy \\ 2 INFM-SMC, Piazzale Aldo Moro 2, I-00185 Rome, Italy and \\ 3 Istituto dei Sistemi Complessi del CNR, Via dei Taurini 19, I-00185, Rome, Italy
}

(Dated: February 2, 2008)

\begin{abstract}
We investigate the effect of electron-phonon interaction on the phononic properties in the onedimensional half-filled Holstein model of spinless fermions. By means of determinantal Quantum Monte Carlo simulation we show that the behavior of the phonon dynamics gives a clear signal of the transition to a charge-ordered phase, and the phase diagram obtained in this way is in excellent agreement with previous DMRG results. By analyzing the phonon propagator we extract the renormalized phonon frequency, and study how it first softens as the transition is approached and then subsequently hardens in the charge-ordered phase. We then show how anharmonic features develop in the phonon propagator, and how the interaction induces a sizable dispersion of the dressed phonon in the non-adiabatic regime.
\end{abstract}

\section{INTRODUCTION}

Despite enormous theoretical efforts, effects arising from the electron-phonon interaction in strongly correlated many-body systems still remain incompletely understood. Achieving an understanding of the complicated interplay between electrons and phonons is, however, essential to explain such diverse phenomena as colossal magnetoresistance in the manganites [1], the Peierls instability in quasi-1D materials [2], and high temperature superconductivity in alkali-metal doped fullerenes 3 . and cuprate compounds [4]. Even in moderately or weakly correlated materials, the electron-phonon interaction can give rise to interesting effects which are not understandable in the framework of the standard theories of electron-phonon interaction, namely the MigdalEliashberg theory of superconductivity and the BornOppenheimer adiabatic principle. As a notable example, we mention the anomalies in the phononic properties of superconducting $\mathrm{MgB}_{2}[\underline{5}]$.

In this work we present a numerical investigation of a simple model for coupled electron-phonon systems the half-filled Holstein model for spinless fermions - and mainly focus on the dynamics of the phononic degrees of freedom. In spite of its apparent simplicity, the model fully accounts for the competition between local quantum fluctuations and the tendency for charge ordering, and is thus a powerful tool to understand the physics of more realistic systems. In one-dimension the system forms a metallic Luttinger liquid for weak electron-phonon coupling. As the coupling is increased, a quantum phase transition occurs to an insulating state with long-range charge-density-wave (CDW) order. A wide variety of methods have been used to investigate this phase transition, with varying degrees of success. Exact diagonalization schemes [6] permit extremely accurate calculation of the ground-state and low-lying excitations, but are limited to treating rather small clusters due to the large Hilbert space required for the phonon degrees of freedom, and are thus subject to large finite-size effects. Larger systems are accessible using the density matrix renormalization group (DMRG) method, and in a recent work 7] Bursill et al were able to determine the location of this phase boundary using this approach. Their result correctly recovers the adiabatic and anti-adiabatic limits (see Fig 1) which can be evaluated analytically [8], and interpolates smoothly between them. Results obtained from a variational Lanczos scheme on small clusters [9] agree well with the DMRG result, but data produced by Quantum Monte Carlo (QMC) methods, such as the worldline QMC method used in the pioneering investigation of Ref. [8] and Green's function QMC [10], show significant deviations, the cause of which is not known. The role of quantum lattice fluctuations on observables has been discussed in Refs. [11, 12], while the spectral properties have been analysed in Refs. 13, 14

In this work we make use of the determinantal QMC method [15] which allows us to compute dynamical properties like the electron and phonon propagators without approximations. These quantities are not equally accessible to DMRG, which is otherwise the best method to establish the phase diagram of one-dimensional models. In contrast to previous QMC investigations we are able to obtain estimates for the location for the phase boundary in excellent agreement with the "benchmark" DMRG result. We concentrate on the phonon propagator, which appears to produce more robust results than the electronic properties considered in previous QMC studies, and show firstly how its behavior reveals the location of the phase-boundary. We then go on to investigate in detail the phonon-softening and anharmonicity effects induced by the electron-phonon interaction by combining analysis of the imaginary-time data with the spectral functions obtained by analytically continuing this data to real frequencies. This allows us to identify a renormalized phonon frequency, and to follow its behavior as a function of electron-phonon coupling. Beside a generic softening of the frequency, we observe how for large bare 


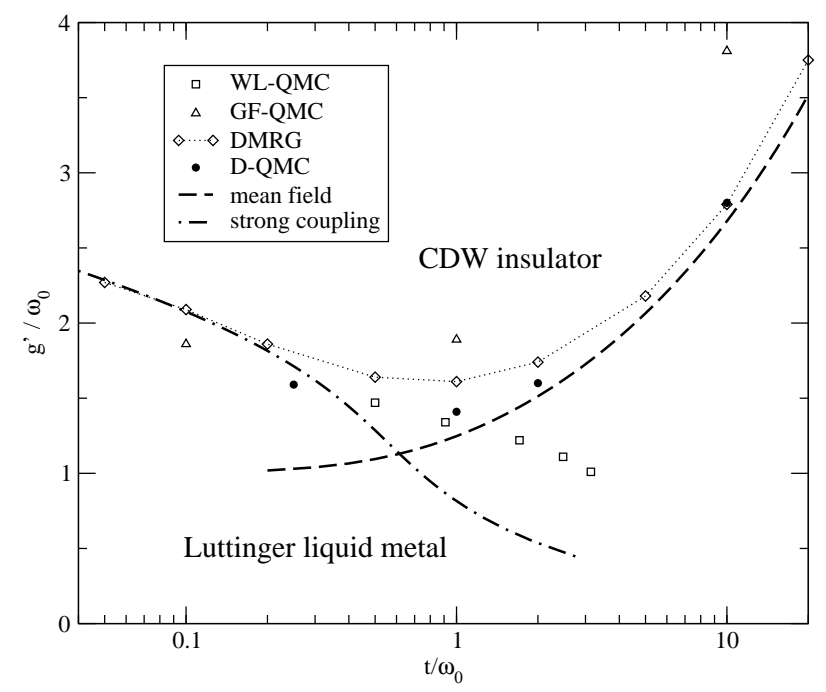

FIG. 1: Phase diagram of the 1D Holstein model, showing the phase boundary between the CDW insulator and Luttinger liquid metal. For ease of comparison with previous results, the $\mathrm{y}$-axis is in units of $g^{\prime}=g / \sqrt{2 m \omega}$, the coupling between the electron and the quantized phonon operators $a_{i} / a_{i}^{\dagger}$. Squares indicate results from the world-line QMC method from Ref. [8], triangles are results from the Green's function QMC investigation of Ref. [10] and the solid circles are the results of this work using determinantal QMC. Diamonds denote the results of the DMRG investigation in Ref. [7].

phonon frequency, the harmonic approximation for the dressed phonon propagator fails as soon as the coupling with the electrons is introduced. We also discuss how the renormalization of the phonon properties also gives rise to a phonon dispersion - even if the bare phonons are dispersionless.

The paper is organized as follows. In Sec. II we introduce the model and some details about the QMC simulations. Sec. III presents the results, and it is in turn divided into two subsections - the phonon propagator in imaginary time and its analytical continuation to real frequencies. In Sec. IV we summarize and give our conclusions.

\section{MODEL AND SIMULATION}

We consider the following Hamiltonian, describing spinless fermions moving on a periodic 1D lattice and interacting with a dispersionless phonon at each lattice site:

$$
\begin{aligned}
H= & -t \sum_{i}\left(c_{i}^{\dagger} c_{i+1}+H . c .\right)-g \sum_{i} n_{i} q_{i}+\mu \sum_{i} n_{i} \\
& +\sum_{i}\left(\frac{p_{i}^{2}}{2 m}+\frac{m \omega_{0}^{2} q_{i}^{2}}{2}\right) .
\end{aligned}
$$

Here $c_{i} / c_{i}^{\dagger}$ are the fermion annihilation/creation operators and $n_{i}$ is the fermion number operator $c_{i}^{\dagger} c_{i}$. The electron-phonon coupling is set by $g$, the phonon frequency is given by $\omega_{0}$, and $q_{i}$ and $p_{i}$ denote the phonon displacement and momentum operators respectively. We will express all energies in units of the electronic hopping $t$, and set the phonon mass $m$ equal to one. Unlike Ref. [8] we work in the grand-canonical ensemble, in which the electronic density is regulated by the chemical potential $\mu$. In this work we only consider the case of the half-filled system, which is given by $\mu=g^{2} / 2 \omega_{0}^{2}$.

To simulate this model we employ the well-known determinantal QMC method (DQMC) developed by Blankenbecler, Scalapino and Sugar [15]. In this approach the fermion degrees of freedom are analytically integrated out of the action, which is straightforward as the Hamiltonian (11) is bilinear in fermion operators, leaving an effective action expressed just in terms of the phonon displacement field. By formally replacing the time coordinate with imaginary-time $(\tau=i t)$, the partition function of the model can then be simulated as a path integral of a Euclidean field theory using standard Monte Carlo techniques. In this formalism the $\tau$-axis represents an additional compact dimension, the extent of which is given by the inverse temperature $\beta$. In order to sample the zero-temperature properties of the system, it is important that sufficiently large values of $\beta$ are used. By comparing the convergence of simulations as $\beta$ was increased we established that in the adiabatic regime $\left(\omega_{0} \leq t\right)$ it was sufficient to take $\beta t=8$, but for the highest phonon frequency that we studied $\left(\omega_{0}=4 t\right)$ a larger value of $\beta t=16$ was required. Increasing the size of $\beta$ also requires increasing the number of time-slices used in the simulation, in order to keep the systematic error arising from the Trotter decomposition sufficiently small. This both increases the simulation's running time and also diminishes its numerical stability, and therefore sets an upper limit on the value of $\omega_{0}$ we are able to treat with this method.

Observables, such as the phonon correlation function, are obtained from the simulation as thermal averages of the form:

$$
\begin{aligned}
D_{i j}(\tau) & =\left\langle q_{i}(\tau) q_{j}(0)\right\rangle, \quad 0 \leq \tau<\beta \\
& =\operatorname{Tr}\left[q_{i}(\tau) q_{j}(0) e^{-\beta H}\right] / Z
\end{aligned}
$$

where $Z=\operatorname{Tr}[\exp (-\beta H)]$, and time-dependent operators are defined as $q(\tau)=\exp (-H \tau) q \exp (H \tau)$. It is important to note that the correlation functions produced by the simulation depend on the imaginary, or Matsubara, time-coordinate $\tau$. To study the system's dynamical properties it is thus necessary to make an analytic continuation of these functions to the real-time domain. This amounts to the solution of the following inverse problem 
16]:

$$
S(\tau)=\int_{-\infty}^{\infty} d \omega \frac{e^{-\omega \tau}}{1+e^{-\beta \omega}} \chi_{T}^{\prime \prime}(\omega)
$$

where $S(\tau)$ is the Matsubara correlation function, and $\chi_{T}^{\prime \prime}(\omega)$ is the imaginary component of the time-ordered susceptibility. To perform this inversion we use a maximum entropy technique [16, 17] based on the singularvalue decomposition 18] of the kernel of Eq[3. By imposing positivity and smoothness constraints on the solution we find that the instabilities typically associated with numerical analytical continuation procedures can be controlled, and this method is thus able to produce stable results of high resolution for the phonon spectral function.

\section{RESULTS}

\section{The Matsubara phonon propagator}

In principle, the transition from the Luttinger liquid phase to the CDW insulator can be observed in the staggered-phonon order parameter

$$
m_{p}=\frac{1}{N} \sum_{j}(-1)^{j}\left\langle q_{j}\right\rangle,
$$

which takes a non-zero value in the CDW phase. It is problematic, however, to measure this quantity directly in a simulation of a finite system, as in the CDW phase the system continually tunnels between the two degenerate ground-states, causing the expectation value of $m_{p}$ to vanish in the ergodic limit. Over short runs, however, a non-zero value can be obtained for $m_{p}$ if the system remains trapped in one of the minima for the duration of the simulation. This allows the location of the phase transition to be located approximately [8], but this estimate is intrinsically rough, and cannot be improved easily as the effect of increasing the number of measurements is to make $m_{p}$ vanish. Nonetheless it provides a useful initial comparison for other estimates, and we found that in all cases it was consistent with the values we obtained by other methods.

As mentioned above, our interest is devoted to the phonon dynamics, which provides us with an alternative way to pinpoint the phase transition. More precisely, we study the phonon propagator (2), which in the noninteracting case $(g=0)$ reads:

$$
D_{i j}^{0}\left(\tau, \omega_{0}\right)=\frac{1}{2 \omega_{0}} \frac{\cosh \omega_{0}(\tau-\beta / 2)}{\sinh \omega_{0} \beta / 2} \delta_{i j} .
$$

It should be noted that as the Holstein phonon is purely local, the bare phonon propagator also only has a trivial spatial dependence. For small electron-phonon coupling it is reasonable to expect that the phonon propagator has the same form, but with with a renormalized (softened) frequency $\Omega$ given by $\Omega^{2} / \omega_{0}^{2}=1-\Pi(0) / \omega_{0}^{2}$, where $\Pi(\omega)$ is the local phonon self-energy in real frequencies. This ansatz is most accurate when the frequency-dependence of the phonon self-energy is weak, and deviations from this ideal behavior are the fingerprints of phonon anharmonicity, i.e. of the difficulty in describing the fully dressed phonon as a single renormalized oscillator.

As the system approaches the CDW transition, the staggered $(k=\pi)$ phonon correlation function is expected to soften and eventually produce a sharp peak at zero frequency, the weight of which measures the condensation of the phonons. For an infinite system at $T=0$, this quantity would be the phonon staggered-order parameter $m_{p}$. On the other hand, the appearance of zerofrequency weight may also occur in the local $(k=0)$ correlation function, which is not, however, directly associated with the CDW transition. In this case, the shift of weight can be associated with a polaron crossover from a good metal to a bad metal, in which the electronic mobility is strongly reduced by the large coupling to the phonons.

We tested this expectation by fitting the local and staggered imaginary time correlation functions with the function $D^{0}(\tau, \Omega)+c$, using the softened frequency $\Omega$ and the shift $c$ as fitting parameters. The rigid shift in imaginary time $c$ is associated with a $\delta$-like peak at zero frequency, and describes the static average of the phonon field. For the local correlator the value of $c$ indicates the static uniform distortion, which reduces the electron mobility through polaronic effects, while for the staggered case it is simply equal to the staggered phonon order parameter $m_{p}^{2}$. For a small value of the bare phononfrequency $\omega_{0}=0.5$, we find that using the above form for the fitting function yields excellent results for both the local and staggered propagators, at all values of the electron-phonon coupling. We show one such example for the staggered propagator in Fig $2 a$. The good quality of the fit is corroborated by the form of the Fourier transform of the correlator, shown to the right. The Fourier representation of the non-interacting propagator (5) can be easily shown to be

$$
D_{i j}^{0}\left(i \omega_{n}, \omega_{0}\right)=\frac{1}{\omega_{0}^{2}+\omega_{n}^{2}}
$$

where $\omega_{n}$ are the Matsubara frequencies, $\omega_{n}=2 \pi n / \beta$. Simply replacing the bare frequency, $\omega_{0}$, in Eq 6 with the renormalized frequency, $\Omega$, obtained from the fitting procedure provides an extremely good fit to the Fourier transform of the Matsubara data, as can be seen in Fig 2b. A similar behavior is observed for $\omega_{0}=1$ (Figs 31, d), where the fitting procedure again yields excellent results for both the Matsubara-time propagator and its Fourier transform. We emphasize that these fits, which essentially pass through every data-point, are ob- 

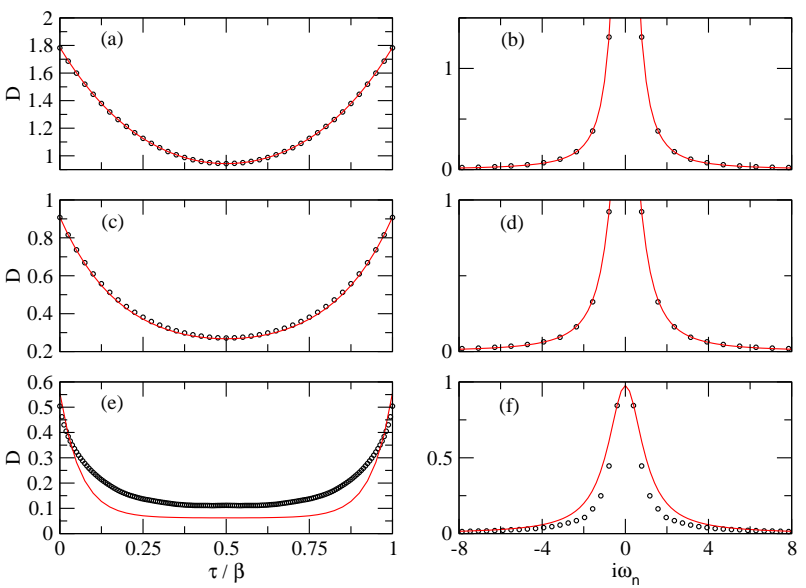

FIG. 2: (a) The staggered phonon propagator as a function of imaginary time for $\omega_{0}=0.5, g=0.6$ (c) for $\omega_{0}=1$ and $g=1.5$ and (e) for $\omega_{0}=4$ and $g=14.0$. All data is taken from simulations of 16 -site systems. The line shows the fit to the form $D\left(\tau, \omega_{0}\right)=D^{0}(\tau, \Omega)+m_{p}^{2}$. In (b), (d) and (f) we compare the corresponding Fourier transforms of these propagators in Matsubara frequency space with the renormalized Lorentzian Eq 6

tained using merely two fitting parameters with a direct and suggestive physical meaning.

On increasing the bare phonon frequency further, however, we find that the fitting function no longer reproduces the data once the electron-phonon coupling is turned on. As can be seen from Fig 2 the curvature of the phonon propagator as a function of $\tau$ cannot be described simply in terms of a softened frequency. This effect can be seen equally distinctly in frequency space in Fig 2, where $D\left(i \omega_{n}\right)$ clearly deviates from the predicted Lorentzian behavior. Effectively the non-adiabatic bare phonon introduces anharmonic effects, which are enhanced by increasing the coupling. The relationship between non-adiabaticity and anharmonicity has been previously proposed for $\left.\mathrm{MgB}_{2} 19\right]$.

In Fig 3 we show the values for the renormalized frequencies, obtained by the fitting procedure, for both the local and staggered phonon propagators. The values of $m_{p}^{2}$ produced by the fitting method are also shown below. For $\omega_{0}=0.5$ (Fig [3] $)$ it can be seen that in the metallic region the local phonon frequencies smoothly reduce from their bare values as $g$ increases, to reach a minimum value at the point $g / \omega_{0}=1.6$. Throughout this process the staggered phonon-frequency is softened to a greater degree than the local phonon. At the local minimum, $m_{p}$ suddenly acquires a non-zero value, indicating that this point signals a transition to the CDW regime, and thus most of the weight in the phonon propagator moves to the zero frequency peak. From Fig 1 it can be clearly seen that this estimate compares extremely well with the
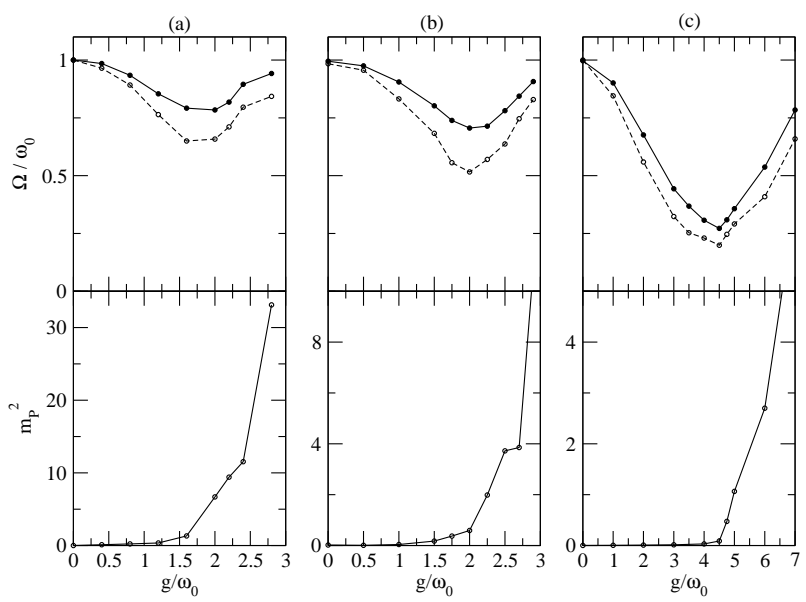

FIG. 3: Softened phonon frequency, $\Omega$, and order parameter, $m_{p}^{2}$, obtained by fitting the phonon propagator to the form $D(\tau)=D^{0}(\tau, \Omega)+m_{p}^{2}$. Solid lines indicate the local phonon frequency, dotted lines the staggered phonon. (a) $\omega_{0}=0.5$, (b) $\omega_{0}=1$ and (c) $\omega_{0}=4$. In all cases the staggered phonon is softened more than the local phonon, and it can be clearly seen that the minimum becomes increasingly deep (i.e. the phonon softens more) as $\omega_{0}$ is increased.

location of the phase transition found in Ref. 7]. On increasing $g$ further, the renormalized frequency is seen to harden [20], and eventually approach the bare frequency in the atomic limit, where the system is no longer metallic and therefore there is no screening of the phonons.

For higher phonon-frequencies a similar behavior occurs, with the softened phonon frequency passing through a well-defined minimum at which $m_{p}^{2}$ turns on. For $\omega_{0}=1$ (Fig[3]) the softening of the phonon frequency is more pronounced, with the staggered phonon frequency decreasing by a factor of one-half as compared with a factor of about two-thirds for the previous case. This trend continues for the antiadiabatic case, $\omega_{0}=4$ (Fig [3:), for which the bare phonon frequency is reduced by almost a factor of ten. Since the fitting procedure is less trustworthy for this case, the values obtained for the fitting parameters should be treated more cautiously. The good agreement with the DMRG result that is nonetheless obtained indicates that the procedure is successfully describing the phonon dynamics with reasonable accuracy, and underlines the reliability of using this behavior as a signal of the phase transition.

Further insight about the effect of the interaction on phononic properties can be gained by the analysis of the statistical distribution of the phonon displacement field $q_{j}$. In Fig. 4 we show how this quantity evolves as the electron-phonon coupling is increased. A clear qualitative change occurs from an unimodal distribution in the metallic phase, in which $q=0$ is the most probable value, 


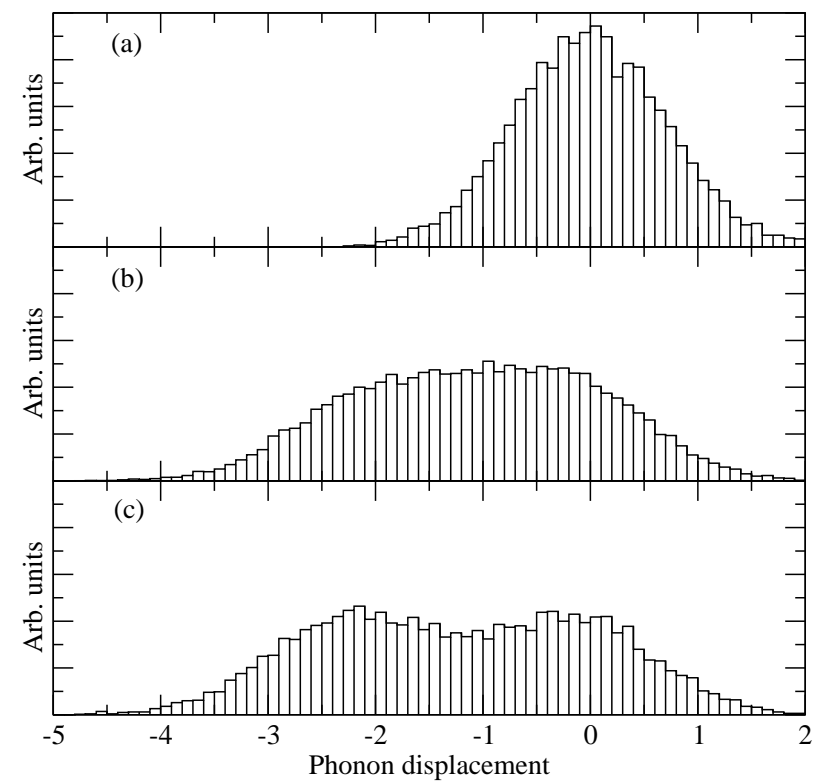

FIG. 4: Histograms of the phonon displacement field $\left\langle q_{i}\right\rangle$ in the vicinity of the phase transition for a system with phonon frequency $\omega_{0}=1$. (a) $g=0.0$, (b) $g=2.25$ and (c) $g=2.5$. Note how the non-interacting Gaussian distribution centered on zero first flattens as the phase transition is approached, and then splits into two in the CDW regime, indicating the formation of a polaronic state.

to a bimodal distribution with maxima 21] separated by $2 q_{0}=g / \omega_{0}^{2}$, which signals the formation of local lattice distortions peaked around $\pm q_{0}$. In previous work [22], the evolution of the distribution of the displacements has been used to characterize the polaron crossover in the Holstein model. In principle, the formation of local distortions does not automatically imply a CDW ordering, as has been discussed for example in Ref. [23]. For the system we study, however, it does appear that the formation of local lattice distortions and CDW ordering do occur simultaneously, implying that a polaronic metal state is not present in the transition region.

\section{Analytic continuation of the phonon propagator}

To complement the previous analysis of the Matsubara correlation functions, we now study the real-frequency propagator by employing a maximum entropy method to make the analytic continuation from Matsubara time to real-frequencies. This avoids the need of assuming a given analytic form for the propagator, and also allows us to study the softening of the phonon in more detail. In Fig [5 we present contour plots of the local phonon spectral function for different bare phonon-frequencies. Darker areas correspond to larger weights. In all figures, the weight is clearly concentrated at the bare frequency $\left( \pm \omega_{0}\right)$ at $g=0$, and for weak coupling it remains concentrated in a single feature at the renormalized frequency $\Omega$. As the renormalized phonon softens further, a zero frequency peak appears and takes most of the weight. In terms of the fitting parameters, the appearance of this feature corresponds to a non-zero value of the shift $c$, implying a sizable static lattice deformation, which reduces the mobility of the electrons. After the CDW transition a higher energy phonon branch forms, and, as was seen previously from the Matsubara analysis, its energy hardens and eventually converges to the bare frequency in the limit of extreme strong-coupling. In Fig 5 the formation of this branch is clearly visible for the non-adiabatic cases $\omega_{0}=1$ and 4 , and although it also occurs in the adiabatic case the higher energy features are rather obscured by the large zero frequency peak. Thus, in the limit of strong-coupling the system recovers the appearance of the atomic limit in which the phonon is unrenormalized, due to the lack of metallic screening.

A similar analysis can be carried out for all the values of the exchanged phonon momentum $k$, resulting in a momentum dependent renormalized phonon frequency $\Omega(k)$. We plot this quantity for $\omega_{0}=1$ and $g=1$ and 2 in Fig. 6] In the weaker coupling case, the renormalized phonon is still basically dispersionless like the bare Holstein phonon. For the larger coupling, however, the renormalized phonon becomes dispersive, exhibiting an approximately $\cos (k / 2)$ dependence on momentum. This dramatically demonstrates that if a material has a sizable electron-phonon interaction, the bare dispersion of the phonons may be substantially different from the fully dressed one. This has to be seriously taken into account in the derivation of effective models, in which the experimentally observed fully dressed dispersion should not be used as a bare dispersion for a model calculation to avoid double counting.

\section{CONCLUSIONS}

In this paper we have investigated the effect of electron-phonon interaction on the half-filled one- 

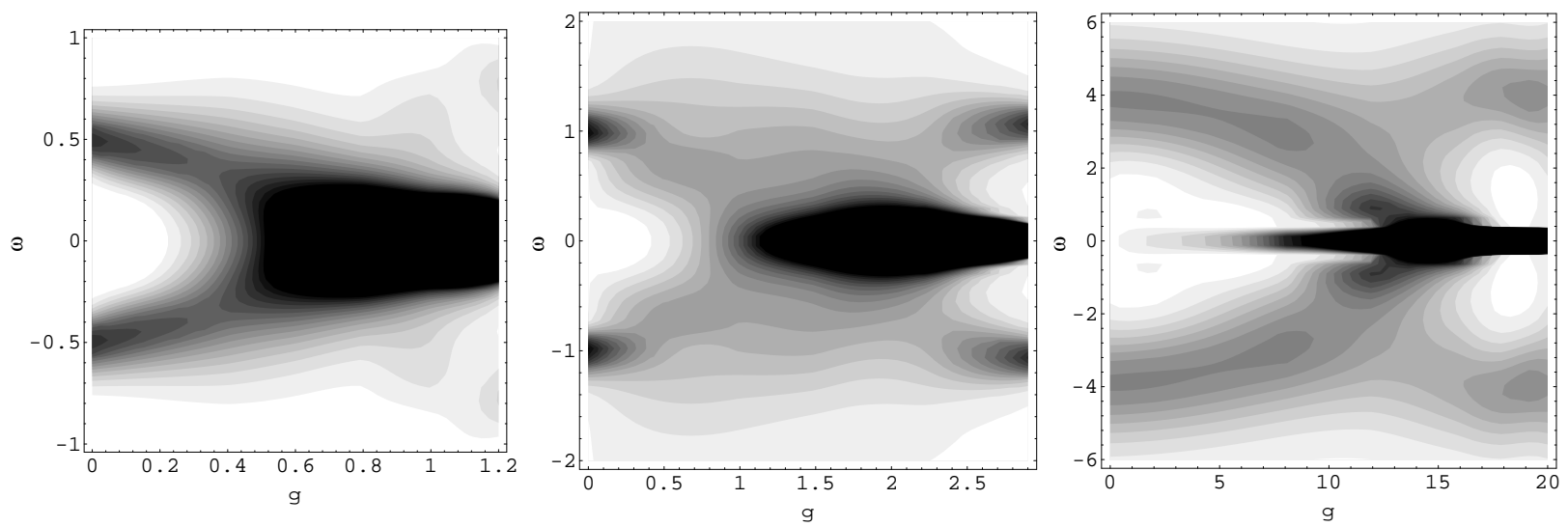

FIG. 5: Analytic continuation of the local phonon propagator $D_{i i}(\tau)$ for phonon frequencies $\omega_{0}=0.5,1.0,4.0$.
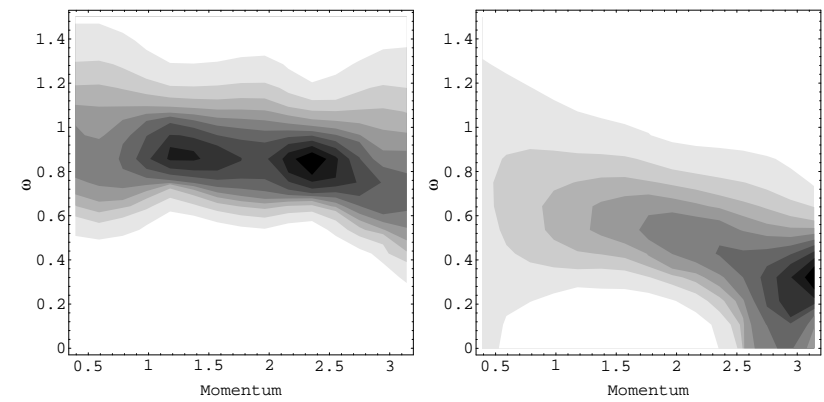

FIG. 6: Phonon susceptibility $\chi^{\prime \prime}(k, \omega)$ for $\omega_{0}=1$. The peaks in this quantity (dark areas) indicate the dispersion relation followed by the dressed phonon. Left: $g=1.0$, the dressed phonon is practically dispersionless, resembling the bare Holstein phonon. Right: $g=2.0$, the phonon peak shifts toward zero as $k$ increases indicating that the dressed phonon has acquired a substantial dispersion from the electron-phonon interaction.

dimensional Holstein model by means of DQMC simulations. We have firstly shown how examination of the phonon propagator can be used to determine the boundary for the CDW phase transition, which agree well with the state-of-the-art DMRG data.

The agreement of our results with the DMRG phase diagram makes us confident about the reliability of our method in the evaluation of other observables, which are more difficult to obtain with DMRG, such as dynamical properties.

In particular, we focus on the phonon propagator, in order to discuss the dressing of the phonon degrees of freedom for large coupling with the electrons. An analysis of the Matsubara phonon propagator allows a renormalized phonon frequency to be deduced directly from the data produced by the QMC simulation. This has revealed how the phonon frequency softens as the coupling is increased until, at a critical value of $g$, the order parameter $m_{p}^{2}$ turns on and the system makes a phase transition to the CDW regime. The degree of softening is considerably higher in the non-adiabatic regime as compared to the adiabatic case. Our analysis has also highlighted the relation between non-adiabatic effects and anharmonicity. When the bare phonon frequency is small, the phonon propagator describes basically a single harmonic phonon even for large coupling, while in the nonadiabatic case, the electron-phonon coupling induces anharmonic effects through a frequency dependent phonon self-energy.

By using analytic continuation methods we were able to study the phonon renormalization in detail. At the critical point we have seen how the phonon mode splits into two - one mode developing into a soft mode, coexisting with the other mode which then hardens and reapproaches the bare frequency as $g$ is increased further. This technique has also revealed the strong momentumdependence of the dressed phonon in the non-adiabatic regime, arising from the electron-phonon interaction. In particular, a sizable phonon dispersion arises as the coupling is increased, even starting from a bare dispersionless phonon, suggesting that care must be taken in building up models for electron-phonon interaction starting from experimentally observed phonon properties.

We thank C. Castellani and S. Ciuchi for valuable discussions. We also acknowledge Italian MIUR Cofin2003 
for financial support.

[1] J. M. De Teresa, M. R. Ibarra, P. A. Algarabel, C. Ritter, C. Marquina, J. Blasco, J. Garcia, A. del Moral, and Z. Arnold, Nature 386, 256 (1997); A. J. Millis, ibid. 392, 147 (1998); M. B. Salamon and M. Jaime, Rev. Mod. Phys. 73, 583 (2001).

[2] Organic Conductors edited by J.-P. Farges, (Marcel Dekker, New York, 1994).

[3] M. Matus, H. Kuzmany, and E. Sohmen, Phys. Rev. Lett. 68, 2822 (1992); K. Harigaya, Phys. Rev. B 45, 13676 (1992); B. Friedman, ibid. 45, 1454 (1992); W. M. You, C. L. Wang, F. C. Zhang, and Z. B. Su, ibid. 47, 4765 (1993).

[4] Guo-Meng-Zhao, M. B. Hunt, H. Keller, and K. A. Muller, Nature 385, 236 (1997); A. Lanzara, P. V. Bogdanov, X. J. Zhou, S. A. Kellar, D. L. Feng, E. D. Lu, T. Yoshida, H. Eisaki, A. Fujimori, K. Kishio, J.-I. Shimoyama, T. Noda, S. Uchida, Z. Hussain, and Z.-X. Shen, ibid. 412, 510 (2001); R. J. McQueeney, J. L. Sarrao, P. G. Pagliuso, P. W. Stephens, and R. Osborn, Phys. Rev. Lett. 87, 77001 (2001).

[5] J. Kortus, I. I. Mazin, K. D. Belashchenko, V. P. Antropov, and L. L. Boyer, Phys. Rev. Lett. 86, 4656 (2001); P. Postorino, A. Congeduti, P. Dore, A. Nucara, A. Bianconi, D. Di Castro, S. De Negri, and A. Saccone, Phys. Rev. B 65, 020507R (2002).

[6] M. Capone, W. Stephan and M. Grilli, Phys. Rev. B 56, 4484 (1997).

[7] Robert J. Bursill, Ross H. McKenzie and Chris J. Hamer, Phys. Rev. Lett 80, 5607 (1998).

[8] Jorge E. Hirsch and Eduardo Fradkin, Phys. Rev. B 27,
4302 (1983).

[9] A. Weiße and H. Fehske, Phys. Rev. B 58, 13526 (1998).

[10] Ross H. McKenzie, C. J. Hamer and D. W. Murray, Phys. Rev. B53, 9676 (1996).

[11] Zhiguo Lü, Qin Wang, and Hang Zheng, Phys. Rev. B 69, 134304 (2004).

[12] C. A. Perroni, V. Cataudella, G. De Filippis, G. Iadonisi, V. Marigliano Ramaglia, and F. Ventriglia, Phys. Rev. B 67, 214301 (2003).

[13] S. Sykora, A. Huebsch, K. W. Becker, G. Wellein, and H. Fehske, Phys. Rev. B 71, 045112 (2005).

[14] Martin Hohenadler, Markus Aichhorn, and Wolfgang von der Linden, Phys. Rev. B 68, 184304 (2003).

[15] R. Blankenbecler, D. J. Scalapino and R. L. Sugar, Phys. Rev. D 24, 2278 (1981).

[16] J. E. Gubernatis, M. Jarrell, R. N. Silver, and D. S. Sivia, Phys. Rev. B 44, 6011 (1991).

[17] R. K. Bryan, Eur. Bio. J. 18, 165 (1990).

[18] C. E. Creffield, E. G. Klepfish, E. R. Pike and S. Sarkar, Phys. Rev. Lett. 75, 517 (1995).

[19] L. Boeri, G. B. Bachelet, E. Cappelluti and L. Pietronero, Phys. Rev. B 65, 214501 (2002).

[20] A. S. Alexandrov, V. V. Kabanov and D. K. Ray, Phys. Rev. B 49, 9915 (1994).

[21] As we do not use an explicitly symmetric form for the electron-phonon interaction term in Eq1(i.e. we use $g q n$ instead of $g q(n-\langle n\rangle))$, this bimodal distribution is not symmetric about the origin, instead having an overall shift of $-q_{0}$.

[22] M. Capone and S. Ciuchi, Phys. Rev. B 65, 104409 (2002).

[23] M. Capone and S. Ciuchi, Phys. Rev. Lett. 91, 186405 (2003). 